Journal of Physics D: Applied Physics, 42(15), 2009. 155402

\title{
Dielectric monitoring of carbon nanotube network formation in curing thermosetting nanocomposites
}

\author{
A Battisti, AA Skordos and IK Partridge \\ Composites Centre, School of Applied Sciences, Cranfield \\ University, Cranfield, Bedfordshire, MK43 0AL, UK \\ E-mail: a.battisti@cranfield.ac.uk
}

\begin{abstract}
This paper focuses on monitoring of carbon nanotube network development during the cure of unsaturated polyester nanocomposites by means of electrical impedance spectroscopy. A phenomenological model of the dielectric response is developed using equivalent circuit analysis. The model comprises two parallel RC elements connected in series, each of them giving rise to a semicircular arc in impedance complex plane plots. An established inverse modelling methodology is utilised for the estimation of the parameters of the corresponding equivalent circuit. This allows a quantification of the evolution of two separate processes corresponding to the two parallel RC elements. The high frequency process, which is attributed to carbon nanotube aggregates, shows a monotonic decrease in characteristic time during the cure. In contrast, the low frequency process, which corresponds to inter-aggregate phenomena, shows a more complex behaviour explained by the interplay between conductive network development and the cross-linking of the polymer.
\end{abstract}

PACS numbers: 73.63.Fg

Submitted to: J. Phys. D: Appl. Phys. 


\section{Introduction}

Recent advances in carbon nanotube (CNT) filled polymer nanocomposites have highlighted the potential of these materials, especially with respect to their electrical properties [1-3]. The formation of percolating networks of high aspect-ratio nanoparticles allows for the development of relatively high conductivities at low concentrations of carbon nanotubes $[4,5]$. A central issue arising when attempting to relate the processing of nanocomposites with the final properties is that of particle dispersion. A variety of dispersion methodologies have been proposed and tested including ultrasonication [6], shear mixing [7, 8], triple roll milling [9] and twin screw extrusion [10]. It is generally believed that a 'good' state of dispersion results in advantageous properties [2]. Some counter-intuitive results such as the occurrence of percolation at loadings significantly lower than expected $[7,11,12]$ or an increase in electrical conductivities observed after the end of dispersion processing $[10,13]$ point to the existence of re-aggregation phenomena that influence the formation of a network of carbon nanotubes.

Dielectric monitoring has been used extensively in the context of thermosetting polymer cure [14-16]. The dielectric response of thermosets is affected by phenomena related to dipolar relaxation and charge migration [17], which are both sensitive to the state of the material. Typically the frequency of dipolar relaxation decreases dramatically during the progress of cure as a result of the process of vitrification, whilst the conductivity due to the movement of mobile charge carriers decreases. The sensitivity of the migrating charge response to the progress of cure forms the basis of quantitative use of dielectric signals. This is carried out in impedance terms via the use of the maximum of imaginary impedance, which is found to correlate strongly with the progress of cure $[18,19]$. The simplification and robustness offered by this form of analysis has now led to the adoption of impedance spectroscopy, coupled with equivalent circuit modelling, in the on-line monitoring of thermoset cure. A further development of this approach, utilised in the present study, was originally developed in solid state physics and electrochemistry [20]; it uses an electrical circuit comprising a set of resistors and capacitors that represent dipolar relaxation, charge migration and electrode polarisation [15] to simulate the impedance response of the curing material. Non-linear fitting of the equivalent circuit response to the experimental data obtained during the cure makes it possible to estimate the circuit element values and their evolution as a function of the progress of the reaction [21]. One limitation 
of using equivalent circuits is the non-uniqueness of the model; the material response can be represented by a variety of combinations of electrical components, all equally fitting the dielectric spectra of the material. The final choice of circuit is usually based on the phenomenological description of the processes involved [22].

Recent work in the authors' group examined the dielectric response of epoxy/CNT nanocomposites during cure and has shown that, when the addition of nanoparticles induces a conductive response, impedance generally increases as cross-linking advances [23]. As in the case of neat thermosets, this behaviour can be attributed to mobility limitations imposed on the charge carriers by the progress of cure [18]. However, in cases where an epoxy matrix has been reinforced with SWNT, the frequency corresponding to the conductive mechanism of the system increases with the progress of cure [24]. This type of behaviour, which has also been observed in thermoplastics filled with nanotubes when melts are allowed to recover from a shear deformation, has been attributed to re-aggregation of carbon nanotubes $[10,13]$. The work reported here focuses on the phenomenon of network formation via re-aggregation in unsaturated polyester/carbon nanotube composites and its influence on the impedance response of the curing material.

\section{Materials and experimental methods}

The resin used was an isophthalic unsaturated polyester (UPE) in a typical formulation that contains inhibitors, and it is cured by the addition of radicalic initiator and a catalyst. This material cross-links by addition polymerisation between polyester chains and the vinyl group of styrene, which in this study was carried out at $40^{\circ} \mathrm{C}$ for 10 hours. Nanocyl $\AA 7000$ multiwalled carbon nanotubes were used in this study. These are nominally 1.5 micrometres long and entangled in rope-like aggregates (figure 1(a-b)), with carbon purity of approximately $90 \%$ [25]. The nanocomposites prepared contains between 0.05 and $0.15 \%$ by weight of filler dispersed by a combination of shear mixing and horn sonication. Details of the preparation procedure are given in a previous publication [26].

Cure kinetics information was gathered using a heat flux differential scanning calorimeter (DSC-TA Instruments 2920). Isothermal cure of the resin was carried out at $40^{\circ} \mathrm{C}$ using sample weights below $10 \mathrm{mg}$. The total heat of reaction was determined from a full cure of the material at $120^{\circ} \mathrm{C}$.

Impedance measurements under thermal profile conditions identical to those 


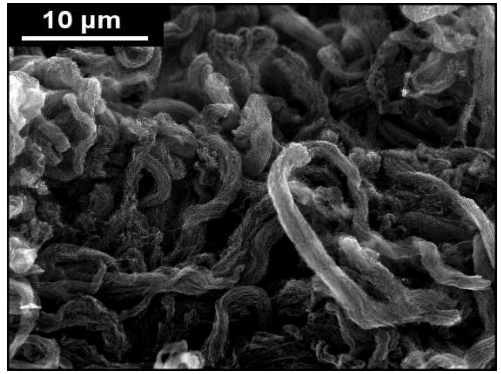

(a)

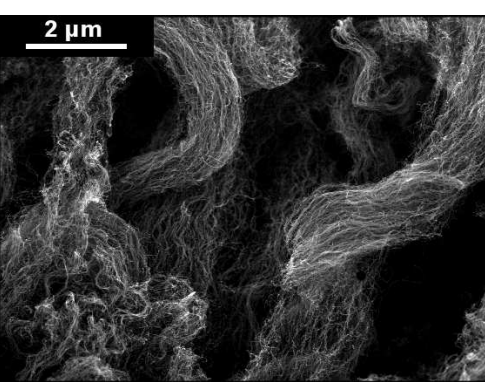

(b)

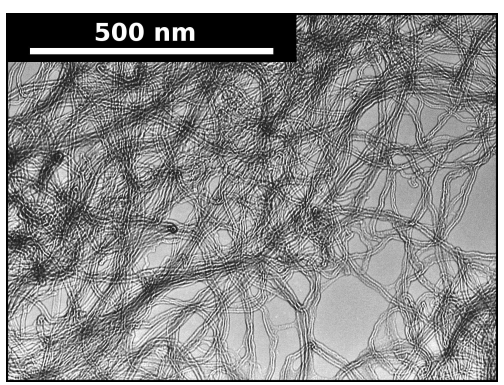

(c)

Figure 1: As-received Nanocyl@8)7000: (a, b) SEM micrograph (c) TEM micrograph

used in the calorimetry tests were performed using a Solartron SI 1260 frequency response analyzer. The instrument communicated with a computer via an IEEEUSB interface. The commercial dielectric sensors used (GIA sensors, Pearson Panke), comprise an assembly of interdigitated copper electrodes, printed with a spacing of $200 \mu \mathrm{m}$ on a thin polymeric film. The sensor was immersed in a glass tube containing the liquid resin and the glass tube was placed in a hollow copper cylinder, surrounded by heating elements controlled by a Eurotherm 2408 temperature controller. A control thermocouple (Type K) was placed in an opening on the wall of the hollow cylinder. A second thermocouple was placed in the glass tube in order to record the actual thermal profile followed by the resin. The measurements were performed in the frequency range between $10 \mathrm{~Hz}$ and $1 \mathrm{MHz}$ for the unfilled resin and between $100 \mathrm{~Hz}$ and $1 \mathrm{MHz}$ for the nanocomposite. Five frequencies per decade were swept on a logarithmic scale.

The morphology of the nanocomposite at low magnifications was investigated by optical transmission microscopy. Specimens of uncured composite were prepared by placing the liquid material between a glass slide and a cover slip and applying pressure to reach a thickness of $50 \pm 10 \mu \mathrm{m}$. Slices of cured material with a similar thickness were cut using a Buehler Isomet precision sectioning saw with a diamond wafering blade. Characterisation of the solid composite morphology at high magnifications was performed on a thin layer of the resin-nanotube dispersion cured on an aluminium stub using a SFEG-SEM (XL-30 Philips/FEI). The analysis was carried out under conditions that induce electron charging of the conductive filler on and to some extent below the surface; this technique is suitable for visualising the network of nanotubes 
embedded in an insulating matrix [27, 28].

\section{Experimental results}

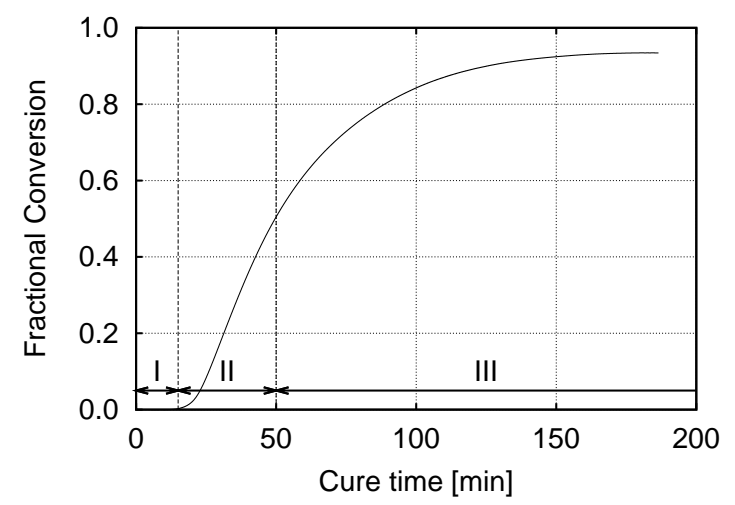

Figure 2: Differential scanning calorimetry results: Degree of cure evolution with time for the isothermal cure at $40^{\circ} \mathrm{C}$, CNT loading $0.10 \mathrm{wt} \%$.

Calorimetry results for the sample containing $0.10 \mathrm{wt} \%$ of carbon nanotubes are illustrated in figure 2. The cure kinetics behaviour is characteristic of a radicalinitiated addition polymerisation. The rate is negligible up to about $15 \mathrm{~min}$ at the cure temperature. This corresponds to the time during which radicals produced are consumed by the inhibitor (stage I in the graph). The reaction speed is maximised at about $50 \mathrm{~min}$ (stage II). After that the reaction slows down significantly and the degree of cure levels off after $150 \mathrm{~min}$ (stage III). The maximum degree of cure reached is $93 \%$, as a consequence of diffusion limitations imposed on the cure reaction after vitrification. 


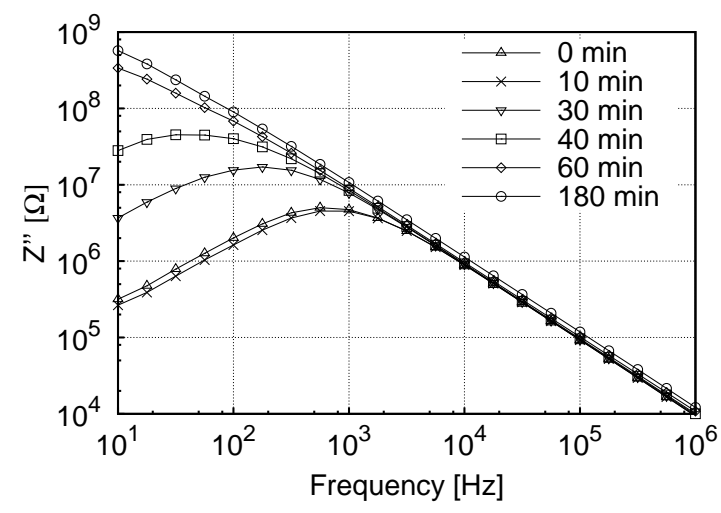

(a)

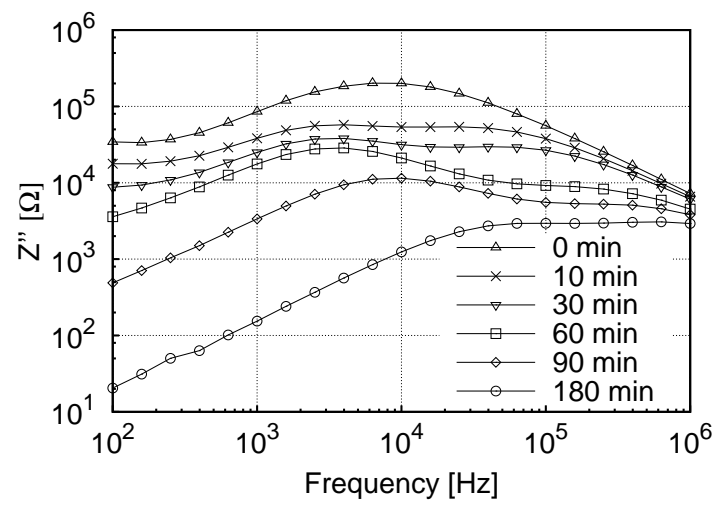

(b)

Figure 3: Evolution of imaginary impedance spectrum during the cure: (a) neat UPE (b) $0.10 \mathrm{wt} \% \mathrm{CNT} / \mathrm{UPE}$ composite.

The results of impedance spectroscopy for the neat resin are illustrated in figure 3(a). The imaginary impedance spectrum is characteristic of the existence of a mechanism of charge migration which is manifested as a peak at intermediate frequencies $[17,18]$. A dipolar relaxation mechanism, which is often manifested as a secondary peak or knee at higher frequencies [18], is also present but cannot be discerned clearly in a logarithm plot. The peak of imaginary impedance starts from a position of approximately $500 \mathrm{~Hz}$ and a level of about $5 \mathrm{M} \Omega$ at the beginning of cure. With the progress of cure the imaginary maximum increases and shifts to lower frequencies, e.g. its value is about $50 \mathrm{M} \Omega$ and its position $20 \mathrm{~Hz}$ after $40 \mathrm{~min}$. After some 60 min the peak moves outside the experimental window of observation. This behaviour is typical of curing thermosets and is attributed to the mobility limitations imposed on charge carriers as the viscosity of the material increases with cross-linking.

The evolution of the imaginary impedance spectrum of the nanocomposite during the cure is illustrated in figure $3(\mathrm{~b})$. The behaviour observed is noticeably different from that of the neat polyester. The initial imaginary impedance spectrum has a peak which is significantly broader than that observed in the neat resin. The peak value decreases by more than one order of magnitude with the addition of nanotubes, as a result of the high conductivity of the filler. Furthermore, the evolution with time is distinctly different in the nanocomposite. The spectrum 
moves towards lower impedance, while the broad peak resolves into two separate peaks. The frequencies of the peaks tend to increase as the cure progresses. Thus, the low frequency peak moves from an initial position of about $2 \mathrm{kHz}$ and a level of approximately $100 \mathrm{k} \Omega$ to a frequency of $10 \mathrm{kHz}$ and a level of $10 \mathrm{k} \Omega$, whilst the high frequency peak shifts from about $10 \mathrm{kHz}$ and $100 \mathrm{k} \Omega$ to $500 \mathrm{kHz}$ and $5 \mathrm{k} \Omega$ after $90 \mathrm{~min}$. This behaviour is indicative of the existence of two mechanisms which are related to the behaviour of the nanoparticles rather than that of the matrix.

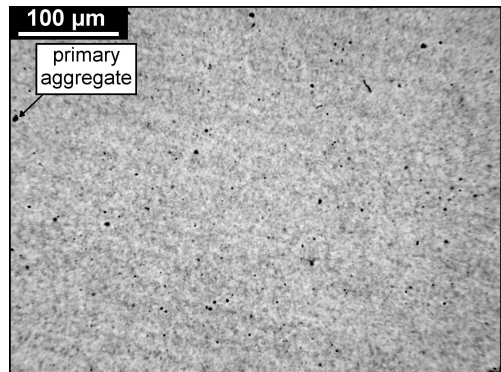

(a)

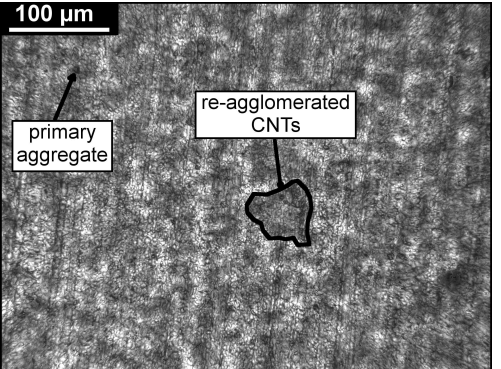

(b)

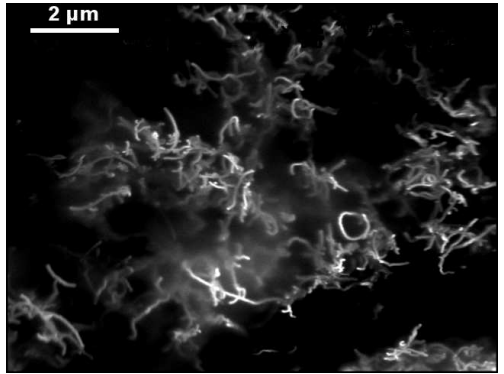

(c)

Figure 4: Micrograph of $0.10 \mathrm{wt} \% \mathrm{CNT} / \mathrm{UPE}$ composite. Optical transmission microscopy of (a) liquid uncured material; (b) fully cured material. (c) Charge contrast imaging SEM of fully cured nanocomposite.

Figures $4(\mathrm{a}-\mathrm{b})$ show the microstructure of the nanocomposite before and after cure, respectively. Prior to cure a slight degree of re-aggregation can be observed. This differs from undispersed primary aggregates, which appear as small dark areas with a size of about 2 to $10 \mu \mathrm{m}$. Some areas are more optically transparent, but the limits of nanotube-rich regions are not well defined. The microstructure of the cured nanocomposite differs significantly, comprising two different types of regions: (i) low transmission areas, which are in the range of 10-100 $\mu \mathrm{m}$, are rich in nanotubes and cover about 50-70\% of the surface; (ii) areas of relatively low nanotube content with high optical transmission. It can be inferred that during the process of cure some process of re-aggregation of initially well dispersed carbon nanotubes occurs. The existence of loose aggregates of carbon nanotubes is evident in contrasting charge scanning electron micrographs of the cured composites, as shown in figure 4(c). 


\section{Modelling of impedance response}

The heterogeneous structure of the nanocomposite observed in microscopy comprises resin-rich regions that are expected to have properties closer to those of neat polyester and filler-rich regions with relatively higher conductivity. The impedance spectra of the system with nanotubes show two mechanisms. These differ significantly from the single mechanism of the neat resin in terms of absolute value, characteristic frequency and evolution during the cure. The impedance response of the composite material is dominated by the behaviour of the conductive loose aggregates of nanotubes and the resin-rich areas acting as interfaces among them. The behaviour of the system can be modelled by the simplest form of the 'bricklayer' equivalent circuit used for the simulation of the behaviour of polycrystalline ceramics $[20,29,30]$. The equivalent

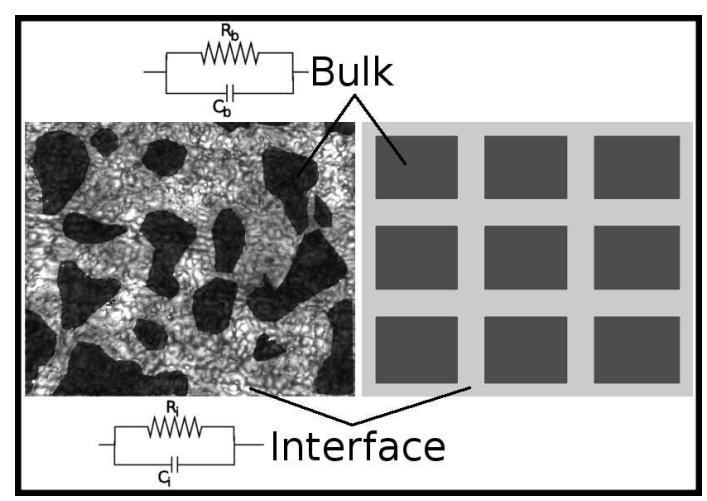

(a)

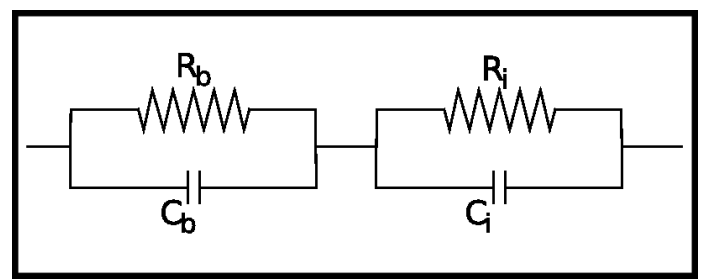

(b)

Figure 5: (a) Schematic of structure of aggregated nanotubes and associated bricklayer model (b) corresponding equivalent circuit.

circuit, illustrated in figure 5(b), comprises two parallel RC sub-circuits connected in series. In the context of the bricklayer model these are assumed to represent the 
bulk resistance and capacitance (b) and the behaviour of interfaces (i). The bulk sub-circuit is expected to result in a relatively higher characteristic frequency, with interfaces occupying the low frequency part of the spectrum.

The impedance of the equivalent circuit is:

$$
\begin{aligned}
Z^{*}(\omega) & =Z^{\prime}(\omega)-j Z^{\prime \prime}(\omega) \\
Z^{\prime}(\omega) & =\frac{R_{b}}{1+\left(\omega C_{b} R_{b}\right)^{2}}+\frac{R_{i}}{1+\left(\omega C_{i} R_{i}\right)^{2}} \\
Z^{\prime \prime}(\omega) & =\frac{\omega C_{b} R_{b}^{2}}{1+\left(\omega C_{b} R_{b}\right)^{2}}+\frac{\omega C_{i} R_{i}^{2}}{1+\left(\omega C_{i} R_{i}\right)^{2}}
\end{aligned}
$$

Here $Z^{*}$ denotes the complex impedance of the circuit, $Z^{\prime}$ and $Z^{\prime \prime}$ are the real and imaginary part of impedance respectively. $C_{b}$ and $R_{b}$ denote the capacitance and resistance of the bulk material, $C_{i}$ and $R_{i}$ are the capacitance and resistance of the interfaces, and $\omega$ is the angular frequency.

The four parameters of the equivalent circuit model are estimated for each set spectrum during the cure of the nanocomposite following the procedure described in [21]. The estimation is performed using a genetic algorithm which minimises the following objective for each frequency sweep:

$$
S\left(R_{b}, C_{b}, R_{i}, C_{i}\right)=\sum_{i=1}^{n} \frac{\left|\bar{Z}\left(\omega_{i}\right)-Z\left(\omega_{i}\right)\right|^{2}}{\left|\bar{Z}\left(\omega_{i}\right)\right|^{2}}, \tau_{b} \leq \tau_{i}
$$

Here $\bar{Z}$ and $Z$ denote the experimental and model impedance respectively, $\omega_{i}$ the frequency of measurement $i$. An additional constraint is used to ensure that the objective function is defined only when the characteristic time corresponding to the bulk sub-circuit $\left(\tau_{b}=R_{b} C_{b}\right)$ is less than the characteristic time of the interfaces subcircuit $\left(\tau_{i}=R_{i} C_{i}\right)$. The introduction of this constraint remedies problems related to the non-uniqueness of solution caused by the use of two identical sub-circuits in the equivalent circuit model. Solutions that are significantly different (more than 20\% in impedance terms) from the previous step are rejected. The estimation is then repeated until a solution within the acceptable limits is found or for a maximum of 50 repetitions. The assumptions underlying this treatment are that values of the parameters obtained in the previous sweep are good a priori estimates for the inverse solution in the current step and that interfacial relaxation occurs at relatively low frequencies. The parameters of the binary genetic algorithm used for the estimation are given in table 1. 
Table 1: Parameters of the genetic algorithm used for the estimation of equivalent circuit parameters.

\begin{tabular}{|c|c|}
\hline Binary string length & 164 digits \\
\hline Number of individuals & 199 \\
\hline Number of generations & 100 \\
\hline Selection operator & roulette wheel \\
\hline Mutation probability & 0.05 \\
\hline
\end{tabular}

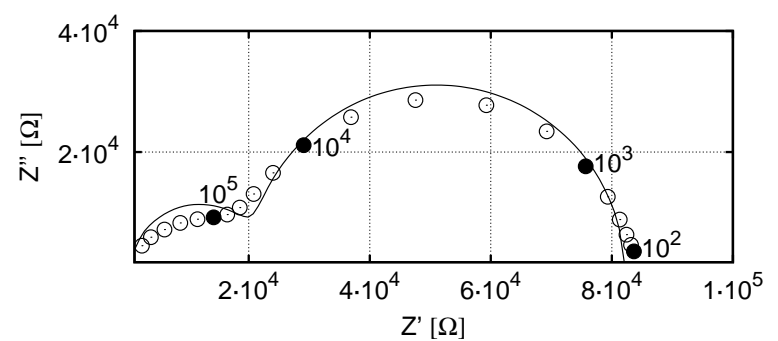

Figure 6: Equivalent circuit analysis of curing nanocomposite impedance response: comparison of experimental and model response at $60 \mathrm{~min}$ in the cure in the form of complex impedance plane plots; O represent the experimental data, solid line represents model results and $\bullet$ denote selected frequencies (given in $\mathrm{Hz}$ ).

Figure 6 illustrates a complex impedance plane plot obtained after about 60 min in the cure. The two semicircles in the experimental data point to the presence of the two mechanisms that are represented by the parallel RC sub-circuits of the model. It can be observed that the two experimental arcs are decentralised and slightly depressed. These are typical of non-Debye type behaviour with distributed relaxation times usually observed in disordered materials and modelled using an equivalent circuit incorporating constant phase elements [20]. The model utilised in this study is limited to an ideal RC behaviour to allow a more robust estimation of the evolution of parameters by minimising the number of parameters and their interrelationships.

The results of parameter estimation are illustrated in figure 7. The evolution of the conductive network can be separated in three different stages, corresponding to the stages of reaction measured by DSC (figure 2). Stage I corresponds to the first 


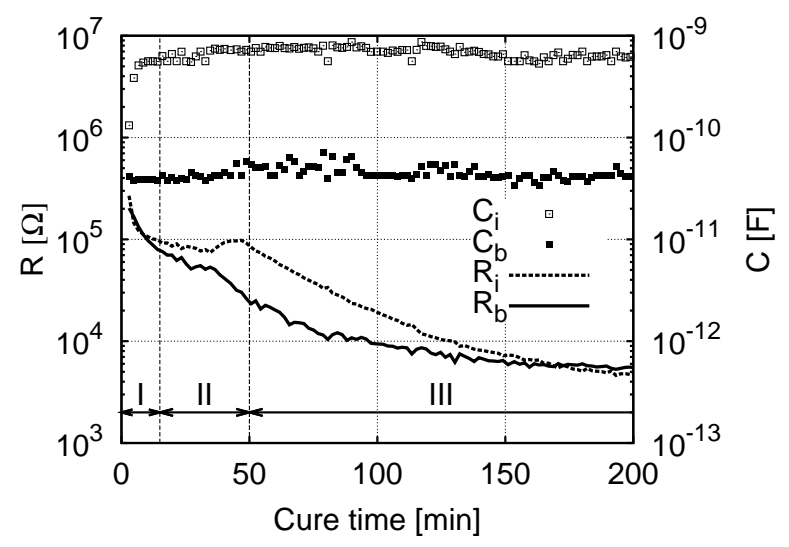

Figure 7: Evolution of the four parameters of the equivalent circuit versus cure time. Composite containing $0.10 \mathrm{wt} \%$ of CNT.

15 minutes of the experiment, during which the degree of conversion is negligible; the interphase shows an increase of capacitance $\left(C_{i}\right)$ and a drop of resistance $\left(R_{i}\right)$, whilst the capacitance of the bulk $\left(C_{b}\right)$ remains approximately constant and the corresponding resistance $\left(R_{b}\right)$ decreases steadily. Stage II corresponds to the time between the onset of curing to approximately the point of maximum rate of reaction; the capacitances $C_{i}$ and $C_{b}$ remain essentially constant, while the behaviour of the two resistances is remarkably different. The two resistances tend to level off at about 35 minutes, at which point they separate: $R_{i}$ increases and $R_{b}$ drops sharply. Reaction continues in stage III, reaching a steady state after about 150 minutes; $C_{i}$ decreases slightly and $C_{b}$ remains constant; $R_{i}$ and $R_{b}$ decay, levelling off at similar final values.

The evolution of characteristic times for composites with different CNT loadings is shown in figure 8. The value of characteristic time at the end of cure is lower at higher filler loading. The characteristic times of the interphase, shown in figure 8(a), start at similar values and then separate during cure. The peak of conductivity due to migrating charges mobility limitations, observed at about 50 minutes at 0.05 wt\%, moves to shorter time and lower intensity with increasing CNT loading. The characteristic times of the bulk phase at the three loadings studied (figure 8b) are well separated from the beginning of cure. 


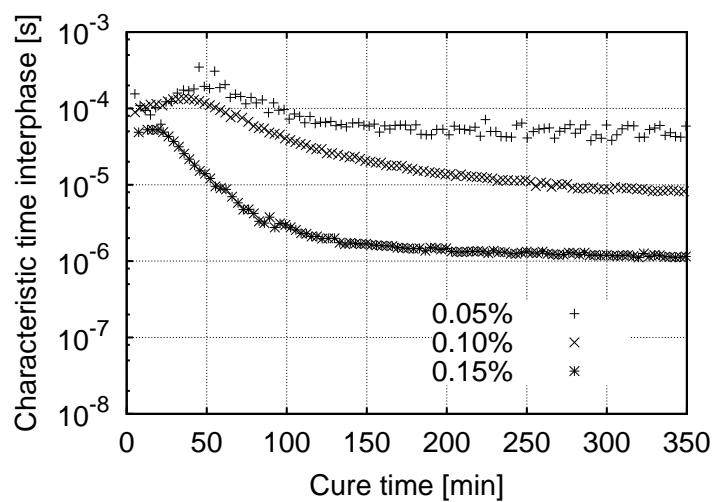

(a)

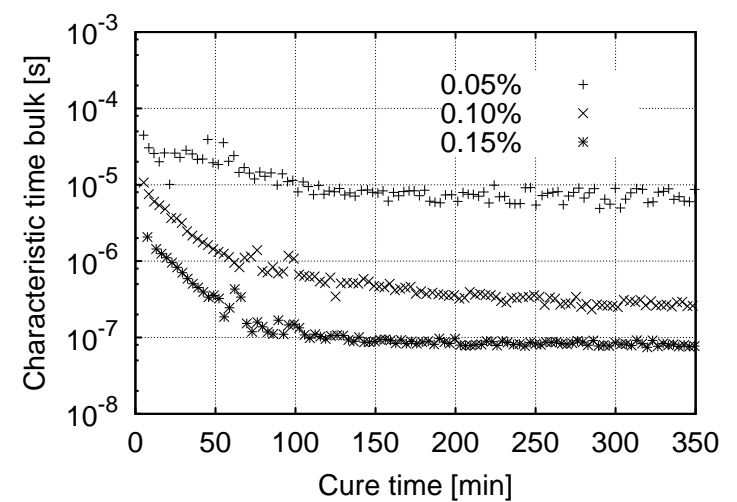

(b)

Figure 8: Comparison of the evolution of characteristic times in CNT/UPE composites at different loadings: (a) interphase between aggregates and (b) bulk of the aggregates.

\section{Discussion}

The results of impedance measurements during the cure of neat polyester and nanocomposites indicate noticeably different behaviours. The neat resin has the response usually observed in curing thermosets, i.e. the existence of a conductive mechanism due to charge migration that is affected by the increasing viscosity of the material as it cures. In contrast, the response of the nanocomposite is dictated by re-aggregation of nanotubes occurring after the end of the process of dispersion. Several models can be used to describe the $\mathrm{AC}$ response of a conductive/insulating matrix composite, such as the effective media Maxwell-Wagner equation, the two exponent phenomenological percolation equation and the bricklayer model [3134]. The bricklayer model with two RC sub-circuits in series, previously used for polycrystalline ceramics [29, 35], has been selected in this study for its ability to separate bulk and interphase contributions and to monitor their changes during cure. The choice is based on the heterogeneous morphology of the nanomaterial observed by microscopy. This morphology is represented schematically in figure 9 .

The evolution of the parameters of the equivalent circuit model during the cure of the nanocomposite is influenced by changes in both the geometry of the conductive network and in the resin properties. The initial sharp increase of the interfacial 


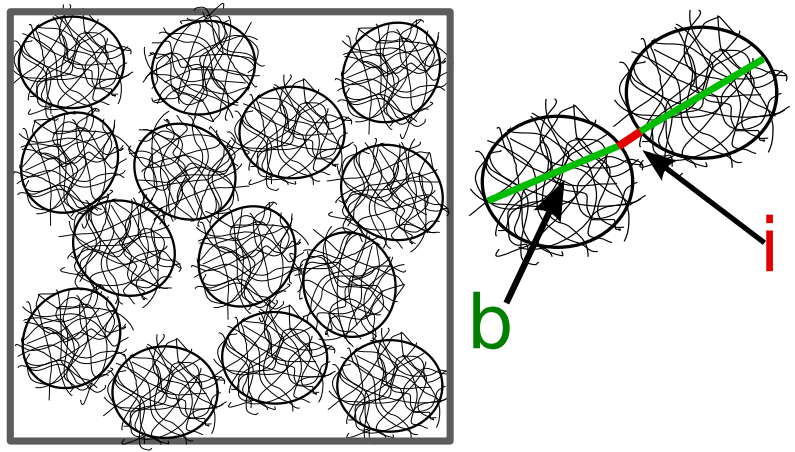

Figure 9: Schematic of carbon nanotube network in insulating thermosetting resin during and after secondary aggregation. Arrows indicate nanotube-rich areas (b) and resin-rich layers (i).

capacitance reflects the generation of interfaces during the formation of aggregates, which in turn become richer in filler, leading to lower resistance. When cure begins, the increase of resin resistivity is manifested as a temporary increase in the resistance of the resin-rich component $\left(R_{i}\right)$. However, the more evident effect of cure on the conductive network is a continuous drop of $R_{b}$ as well as $R_{i}$ during the progress of reaction, leading to final values of more than one order of magnitude lower than those at the beginning of cure.

The values of resistance and capacitance of the equivalent circuit are influenced by geometrical factors as well as by material properties. Calculation of the characteristic time corresponding to each of the sub-circuits allows elimination of some of the effects related to geometry. Figure 8 shows the evolution of the characteristic time of the two sub-circuits during the cure. The sequence of the three stages described previously is repeated for all loadings, although at the lower loading scatter is higher due to the low signal/noise ratio caused by the low conductivity of the material. The characteristic time of interfaces $\left(\tau_{i}\right)$ is about one order of magnitude higher than that of the bulk $\left(\tau_{b}\right)$ for all loadings. The characteristic time of the interface sub-circuit is almost constant during the first part of cure although both the resistance and capacitance change significantly. This fact points to a pure geometrical effect occurring in the first stages of cure, during which new interface is produced. Subsequently, the characteristic time increases slightly as a result of rising mobility limitations imposed by polymerisation of the thermosetting matrix. 
After this point, the characteristic times of both interfaces and bulk decay until the end of the cure, indicating a contribution from the reaction to the resistivity of both phases.

The characteristic times at the end of cure of both interphase and bulk decrease with carbon nanotube content. This is in agreement with the expected increase of conductivity with filler loading, typical of percolating systems. The peak of conductivity due to migrating charges mobility limitations is less evident at higher loadings, as shown in figure $8(\mathrm{a})$. This is due to the faster drop of interphase resistance, which causes the shifting and hindering of the peak. The separation of characteristic times of the bulk phase at the three loadings (figure 8b) suggests the existence of a different conductivity of the loose aggregates of carbon nanotubes. The decay of characteristic time of both components suggests that reaction affects the electrical properties of the composite, improving its conductivity. Although this effect is observed on both the bulk of the CNT aggregates and the resin-rich interphase, the latter is more significant at higher loading.

\section{Conclusions}

The investigation of the dielectric behaviour during cure of unsaturated polyester/carbon nanotube composites highlighted significant differences between the response of nanocomposites and the thermosetting matrix. The imaginary impedance spectrum of the nanocomposite shows two mechanisms, both moving to lower impedance and higher frequencies with the progress of cure. This behaviour is attributed to the re-aggregation of carbon nanotubes observed after the end of dispersion processing. This aggregation leads to an heterogeneous morphology of the nanocomposite, with nanotube rich regions of size in the range of 10-100 $\mu \mathrm{m}$. The heterogeneous morphology can be modelled by an equivalent circuit comprising two parallel RC sub-circuits, one of them representing the nanotube rich regions and the second the interphase that surround them. Parameter estimation based on a genetic algorithm was used to estimate the evolution of the parameters of the circuit during the cure. This analysis was capable of evaluating the influence of material properties changes as well as of geometrical characteristics of the morphology. The generation of interphase induced by re-aggregation affects both elements of the corresponding sub-circuit, whilst the drop in conductivity of the thermosetting matrix influences the resistance of interfaces. The phenomenon of aggregation causes an increase in 
conductivity in the nanotube rich regions, which is reflected in a drop in the resistance of the bulk sub-circuit. The characteristic times of the two sub-circuits follow closely the local material response rather than geometrical effects. The characteristic time corresponding to the nanotube rich regions decreases continuously, whilst that of interphase is non-monotonous due to the interplay between the changes in the electrical properties of the resin and the effect of cure on the carbon nanotubes. It follows the behaviour of resin, with an increasing trend up to a point which reverses in the later stage of cure. At higher filler loadings the effect of the nanotubes is dominant and only a decay can be seen.

The information obtained using this type of analysis can form the basis for on-line monitoring of dispersion/re-aggregation in the context of nanocomposites processing. The phenomenological model reported in this study can facilitate the understanding of the changes of electrical characteristics of a nanocomposite during cure, which in turn determine its final electrical conductivity. The contributions of the different components can be discerned, which can be a useful tool for control and optimisation of the cure of nanocomposites. Implementation in on-line process monitoring, e.g., RTM or pultrusion, could be applied as quality control for the industrial production of fibrous composites containing a nanocomposite matrix.

\section{Acknowledgments}

The authors would like to thank Dr. S Dunn and Dr. D Gallardo for valuable discussions. Finance from EPSRC (EP/E001874 and EP/C509730) and donation of materials from Scott Bader Ltd are gratefully acknowledged.

\section{References}

[1] M. Moniruzzaman and K. I. Winey. Polymer nanocomposites containing carbon nanotubes. Macromolecules, 39(16):5194-5205, 2006.

[2] E. T. Thostenson, Z. Ren, and T. W Chou. Advances in the science and technology of carbon nanotubes and their composites: A review. Composites Science and Technology, 61(13):1899-1912, 2001.

[3] R. H. Baughman, A. A. Zakhidov, and W. A. De Heer. Carbon nanotubes - the route toward applications. Science, 297(5582):787-792, 2002. 
[4] W. Bauhofer and J. Z. Kovacs. A review and analysis of electrical percolation in carbon nanotube polymer composites. Composites Science and Technology, 2008. In Press.

[5] J. N. Coleman, S. Curran, A. B. Dalton, A. P. Davey, B. McCarthy, W. Blau, and R. C. Barklie. Percolation-dominated conductivity in a conjugated-polymercarbon-nanotube composite. Physical Review B - Condensed Matter and Materials Physics, 58(12):R7492-R7495, 1998.

[6] D. Qian, E. C. Dickey, R. Andrews, and T. Rantell. Load transfer and deformation mechanisms in carbon nanotube-polystyrene composites. Applied Physics Letters, 76(20):2868-2870, 2000.

[7] J. Z. Kovacs, B. S. Velagala, K. Schulte, and W. Bauhofer. Two percolation thresholds in carbon nanotube epoxy composites. Composites Science and Technology, 67(5):922-928, 2007.

[8] A. Moisala, Q. Li, I. A. Kinloch, and A. H. Windle. Thermal and electrical conductivity of single- and multi-walled carbon nanotube-epoxy composites. Composites Science and Technology, 66(10):1285-1288, 2006.

[9] F. H. Gojny, M. H. G. Wichmann, B. Fiedler, I. A. Kinloch, W. Bauhofer, A. H. Windle, and K. Schulte. Evaluation and identification of electrical and thermal conduction mechanisms in carbon nanotube/epoxy composites. Polymer, 47(6):2036-2045, 2006.

[10] S. Pegel, P. Potschke, G. Petzold, I. Alig, S. M. Dudkin, and D. Lellinger. Dispersion, agglomeration, and network formation of multiwalled carbon nanotubes in polycarbonate melts. Polymer, 49(4):974-984, 2008.

[11] J. K. W. Sandler, J. E. Kirk, I. A. Kinloch, M. S. P. Shaffer, and A. H. Windle. Ultra-low electrical percolation threshold in carbon-nanotube-epoxy composites. Polymer, 44(19):5893-5899, 2003.

[12] M. B. Bryning, M. F. Islam, J. M. Kikkawa, and A. G. Yodh. Very low conductivity threshold in bulk isotropic single-walled carbon nanotube-epoxy composites. Advanced Materials, 17(9):1186-1191, 2005.

[13] I. Alig, T. Skipa, D. Lellinger, and P. Potschke. Destruction and formation of a carbon nanotube network in polymer melts: Rheology and conductivity spectroscopy. Polymer, 49(16):3524-3532, 2008. 
[14] S. D. Senturia and N. F. Sheppard. Dielectric analysis of thermoset cure. Advances in Polymer Science, 80:1-47, 1986.

[15] J. Mijovic, J. M. Kenny, A. Maffezzoli, A. Trivisano, F. Bellucci, and L. Nicolais. The principles of dielectric measurements for in situ monitoring of composite processing. Composites Science and Technology, 49(3):277-290, 1993.

[16] George M. Maistros and Ivana K. Partridge. Dielectric monitoring of cure in a commercial carbon-fibre composite. Composites Science and Technology, 53(4):355-359, 1995.

[17] S. Andjelic, J. Mijovic, and F. Bellucci. Impedance spectroscopy of reactive polymers. 5. impedance as a measure of chemical and physical changes in glass formers. Journal of Polymer Science, Part B: Polymer Physics, 36(4):641-653, 1998.

[18] J. Mijovic and C. F. W. Yee. Use of complex impedance to monitor the progress of reactions in epoxy/amine model systems. Macromolecules, 27(25):7287-7293, 1994.

[19] A. A. Skordos and I. K. Partridge. Determination of the degree of cure under dynamic and isothermal curing conditions with electrical impedance spectroscopy. Journal of Polymer Science, Part B: Polymer Physics, 42(1):146$154,2004$.

[20] J. R. Macdonald. Impedance Spectroscopy. Emphasizing Solid Material and Systems. John Wiley and Sons, New York, 1987.

[21] M. C. Kazilas, A. A. Skordos, and I. K. Partridge. Parameter estimation in equivalent circuit analysis of dielectric cure monitoring signals using genetic algorithms. Inverse Problems in Science and Engineering, 13(2):157-176, 2005.

[22] J. Mijovic. Dielectric spectroscopy of reactive network-forming polymers, chapter 9. Broadband Dielectric Spectroscopy. Springer, first edition, 2002.

[23] A. Dimopoulos, A. A. Skordos, and I. K. Partridge. Cure of a carbon nanotube modified multiphase epoxy- thermoplastic resin system. In 49th AIAA/ASME/ASCE/AHS/ASC Structures, Structural Dynamics, and Materials Conference, Schaumburg, IL, USA, 7 - 10 April 2008 2008. American Institute of Aeronautics and Astronautics.

[24] L. Valentini, D. Puglia, E. Frulloni, I. Armentano, J. M. Kenny, and S. Santucci. 
Dielectric behavior of epoxy matrix/single-walled carbon nanotube composites. Composites Science and Technology, 64(1):23-33, 2004.

[25] Nanocyl 7000 data sheet. www.nanocyl.com/products/industrial/7000.php.

[26] A. Battisti, A. A. Skordos, and I. K. Partridge. Monitoring dispersion of carbon nanotubes in a thermosetting polyester resin. Composites Science and Technology, 2008. In Press.

[27] J. Z. Kovacs, K. Andresen, J. R. Pauls, C. P. Garcia, M. Schossig, K. Schulte, and W. Bauhofer. Analyzing the quality of carbon nanotube dispersions in polymers using scanning electron microscopy. Carbon, 45(6):1279-1288, 2007.

[28] J. Loos, A. Alexeev, N. Grossiord, C. E. Koning, and O. Regev. Visualization of single-wall carbon nanotube (swnt) networks in conductive polystyrene nanocomposites by charge contrast imaging. Ultramicroscopy, 104(2):160-167, 2005.

[29] P. Heitjans and S. Indris. Diffusion and ionic conduction in nanocrystalline ceramics. Journal of Physics Condensed Matter, 15(30):R1257-R1289, 2003.

[30] J. T. S. Irvine, D. C. Sinclair, and A. R. West. Electroceramics : Characterization by impedance spectroscopy. Advanced Materials, 2(3):132-138, 1990.

[31] D. S. McLachlan, J. H Hwang, and T. O. Mason. Evaluating dielectric impedance spectra using effective media theories. Journal of Electroceramics, $5(1): 37-51,2000$.

[32] G. Sauti and D. S. McLachlan. Impedance and modulus spectra of the percolation system silicon-polyester resin and their analysis using the two exponent phenomenological percolation equation. Journal of Materials Science, 42(16):6477-6488, 2007.

[33] D. S. Mclachlan. Analytical functions for the dc and ac conductivity of conductor-insulator composites. Journal of Electroceramics, 5(2):93-110, 2000.

[34] D. S. McLachlan, C. Chiteme, C. Park, K. E. Wise, S. E. Lowther, P. T. Lillehei, E. J. Siochi, and J. S. Harrison. Ac and dc percolative conductivity of single wall carbon nanotube polymer composites. Journal of Polymer Science, Part B: Polymer Physics, 43(22):3273-3287, 2005.

[35] S. K. Kim, M. Miyayama, and H. Yanagida. Electrical anisotropy and a plausible 
explanation for dielectric anomaly of bi4ti3o12 single crystal. Materials Research Bulletin, 31(1):121-131, 1996. 